\title{
Non-communicable Diseases and COVID-19; a double-edged sword A Special Communication from IRAN
}

\author{
Niloofar Peykari ${ }^{1}$ (1) - Sana Eybooosh ${ }^{2}$ (1) - Hamidreza Safikhani ${ }^{3} \cdot$ Ali Akbar Haghdoost $^{4,5}$ (D) \\ Ozra Tabatabaei-Malazy ${ }^{6,7}$ (D) $\cdot$ Bagher Larijani $^{7,8}$ (1)
}

Received: 7 October 2020 / Accepted: 12 October 2020 / Published online: 23 November 2020

(C) Springer Nature Switzerland AG 2020

\section{Introduction}

The novel coronavirus disease (COVID-19) affected $8,366,417$ people, and caused 450,087 deaths over the time up to 19 June 2020 [1]. Nearly 856,650 confirmed cases of covid-19 were related to Eastern Mediterranean region [1]. The COVID-19 crisis affected Iran similar to other countries in the world and region, and 184,955 confirmed cases reported till 13 June 2020 in Iran [2].

In recent decades, non-communicable diseases (NCDs) have been the most cause of death in the world [3]. In global level, $73.4 \%$ of total death occurred due to NCDs in 2017, and $82.3 \%$ of peoples in Iran died from NCDs according to the latest information of global burden of disease (GBD) 2017 [4].

Now, by dramatic surge of novel coronavirus disease in the world, we faced to double failure situation. Not only, NCDs could be led to risk of COVID-19 severity, but also COVID19 could be worse comorbidities' situation [5]. A systematic

Bagher Larijani

emrc@tums.ac.ir

Niloofar Peykari

niloofarpeykari@gmail.com

Sana Eybpoosh

sana.eybpoosh@gmail.com

Hamidreza Safikhani

safikhani.hr@gmail.com

Ali Akbar Haghdoost

ahaghdoost@gmail.com

Ozra Tabatabaei-Malazy

tabatabaeiml@sina.tums.ac.ir

1 Iranian non-communicable disease committee, and national committee for COVID-19 epidemiology, Ministry of Health and Medical Education, Tehran, Iran review on the prevalence of comorbidities in the confirmed COVID-19 patients revealed that hypertension (21\%), diabetes mellitus (11\%), cardiovascular disease $(5.8 \%)$, chronic kidney disease $(3.6 \%)$, malignancy $(2.7 \%)$, cerebrovascular disease $(2.4 \%)$, chronic pulmonary disease $(2.0 \%)$ were considerable [5].

A study on the clinical characteristics of COVID-19 in Taizhou, China showed that, severely ill patients compare with non-sever patients had a higher proportion of diabetes mellitus ( $16.3 \%$ vs. $6.9 \%$ ), and had a higher body mass index (mean, 24.78 vs. $23.20 \mathrm{~kg} / \mathrm{m}^{2}$ ) [6]. Also a retrospective case series study in China on clinical characteristics of died patients with covid-19 showed chronic hypertension and other cardiovascular comorbidities were more in deceased patients compare with recovered patients, $48 \%$ vs $14 \%$, and $24 \%$ vs $4 \%$, respectively [7].

Therefore, we aimed to provide an overview on the main NCDs and COVID-19 to better understand the situation for

2 Department of Epidemiology and Biostatistics, Research Centre for Emerging and Reemerging Infectious Diseases, Pasteur Institute of Iran, Tehran, Iran

3 Information Technology Center, Ministry of Health and Medical Education, Tehran, Iran

4 Education, COVID-19 Epidemiology, Ministry of Health and Medical Education, Tehran, Iran

5 Modeling in Health Research Center, Institute for Futures Studies in Health, Kerman University of Medical Sciences, Kerman, Iran

6 Non-Communicable Diseases Research Center, Endocrinology and Metabolism Population Sciences Institute, Tehran University of Medical Sciences, Tehran, Iran

7 Endocrinology and Metabolism Research Center, Endocrinology and Metabolism Clinical Sciences Institute, Tehran University of Medical Sciences, No.10, Next to Dr. Shariati Hospital, Jalal Al Ahmad Hwy., North Kargar Ave., Tehran 1411713119, Iran

8 Iranian National Committee for NCDs Prevention and Control, Ministry of Health and Medical Education, Tehran, Iran 
better responsiveness in future and present a feature of Iran in this regards.

\section{Cardiovascular diseases}

According to a meta-analysis, the pooled prevalence of hypertension, and cardiovascular disease in people infected with novel corona virus-2019 were estimated as $16.37 \%(95 \%$ CI: 10.15-23.65), and 12.11\% (95\% CI: 4.40-22.75), respectively [8]. Interaction between cardiovascular diseases (CVD) and COVID-19 need to especial management. A review article claimed acute cardiac injury was the most common reported cardiac abnormality (8-12\%) in COVID-19. Nevertheless, the history of CVD in COVID-19 patients was associated with worse outcome [9-11].

Among 44,672 individuals confirmed cases of COVID-19 in china, $12.8 \%$ had hypertension and $4.2 \%$ had CVD. Patients hospitalized with COVID-19 who had CVD were more suffered from severe illness, and had a much higher fatality rate [12]. Therefore, specific attention should be given to cardiovascular protection during the COVID-19 treatment [10].

\section{Diabetes}

A meta-analysis estimated pooled prevalence of diabetes in people infected with COVID-19 as $7.87 \%$ (95\% CI: $6.57-$ 9.28) [8]. Diabetes has been an important risk factor for pneumonia, and mortality in patients infected Influenza A (H1N1), Severe Acute Respiratory Syndrome (SARS), and Middle East Respiratory Syndrome-related Coronavirus (MERS$\mathrm{CoV}$ ) [13]. Prolonged uncontrolled hyperglycemia may be important in the pathogenesis of the disease [14]. It is considerable, Interleukin-6 (IL-6) was significantly higher in diabetic patients than non-diabetic people. It increased in chronic inflammation, and it may play a more deleterious role in COVID-19 [15].

A meta-analysis showed the diabetic patients have significantly increased risk of emerging severe COVID-19 compared to non-diabetic patients, (OR: 2.61; 95\% CI: 2.053.33) [16]. In addition, a report of 72,314 cases of COVID19 published by china showed an increasing mortality rate in diabetic peoples than other cases infected COVID-19 (7.3\% vs $2.3 \%$ ) [17].

In diabetic patients, glycemic control could be benefit for reducing the risk of infection and severity of COVID-19. China recommended subcutaneous insulin injections for patients with mild COVID-19, and preferably, intravenous insulin therapy for severe COVID-19 patients [18].

\section{Cancer}

According to the Italian Medical Council, $16.5 \%$ of 909 patients who died from COVID-19 had cancer [19]. A meta- analysis estimated the pooled prevalence of cancer in COVID-19 patients $2.0 \%$ (95\% CI: 2.0-3.0) [20]. In another study was shown that patients with cancer in older age had worse outcomes from COVID-19 [21].

Cancer has been reported as an important risk factor for adverse outcomes of COVID-19. It seems patients with cancer have more severe COVID-19 symptoms than others [22]. Importantly, the hazard ratio (HR) of severe events such as the need to invasive ventilation or death was $39 \%$ in patient with cancer and infected COVID-19compare with persons without cancer $(8 \%)$. Moreover, the limited data revealed a higher risk of clinically severe events for COVID-19 cases that suffered from cancer and underwent chemotherapy or surgery in the past month (75\% vs 43\%) [20]. The other study showed in COVID-19 patients with cancer who receiving the most recent cancer treatment within 14 days, the hazard ratio (HR) of severe clinical events was 4.1, (95\% CI: 1.09-15.32; $P=0.037$ ) [22]. Therefore, the more intensive surveillance or treatment should be considered in cancer patients who infected with SARS-coronavirus 2 (SARS-CoV-2) [23].

\section{Chronic respiratory disease (CRD)}

Chronic Obstructive Pulmonary Disease (COPD) had a significant impact on poor outcome of covid-19 that reflected by a HR of 2.681 (95\% CI: 1.424-5.0480) after adjusting for age and smoking status. The ongoing symptomatology of COPD leads to delayed diagnosis of COVID-19, and could be led to respiratory failure [24].

The pooled Odds ratio of COPD and the development of severe COVID-19 was reported 4.38 (fixed-effects model; 95\% CI: 2.34-8.20) in a study. Their results indicate that pre-existing COPD probably worsen the prognosis of COVID-19 [25]. COPD patients have been more at risk of the severity the novel coronavirus disease; $63 \%$, (22/35) compared to patients without COPD 33.4\% (409/1224), and there was association with higher mortality (60\%) [26].

Patients with asthma and COPD are recommended to closely adhere to their prescribed inhaler medication therapy [27].

\section{Comorbidities of main NCDs with COVID-19 in hospitalized confirmed cases of Iran}

According to daily situation report of Iran on March 23, 2020, $27.8 \%$ of the lab-confirmed infected cases and $33.3 \%$ of the death cases had at least one co-morbidity [28]. Based on the latest report in this regards on May 12, 2020, cases with at least one co-morbidity was $30.2 \%$, and the percent of COVID19 died cases who had at least one co-morbidity was $37.9 \%$ (corona.behdasht.gov.ir). 
Fig. 1 Relative Frequency of the Main NCDs as comorbidities in hospitalized confirmed cases of COVID-19. $C V D$ cardiovascular diseases, $C R D$ Chronic respiratory diseases

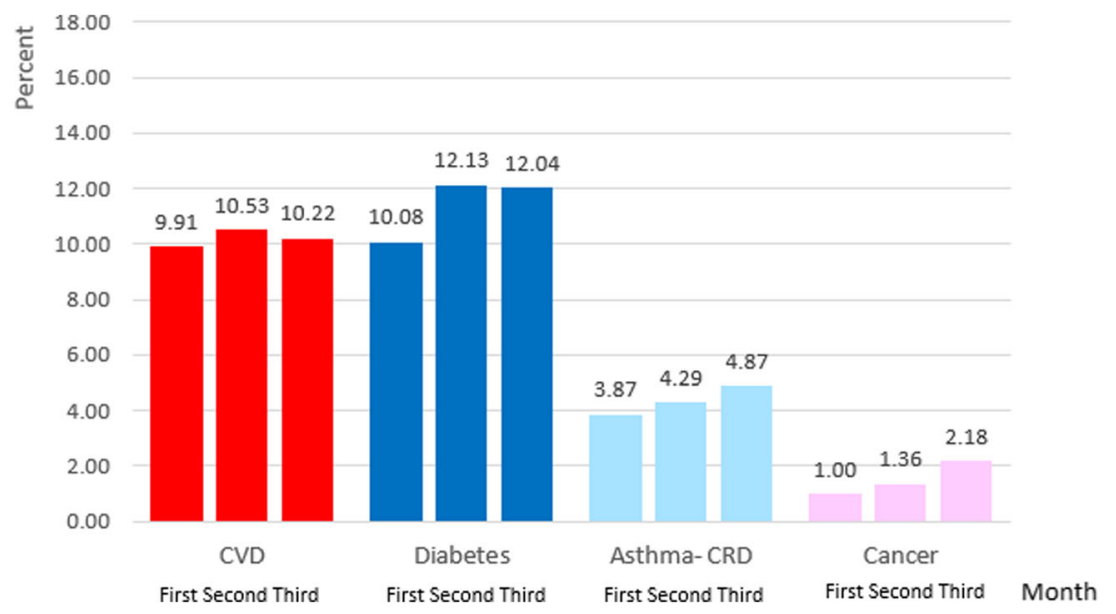

From Feb 20, 2020 to May 20, 2020, the analysis of medical care monitoring center (MCMC) data showed diabetes had the most relative frequency among hospitalized confirmed cases of COVID-19, and it is increased from $10.08 \%$ to $12.04 \%$ across 3 months. Moreover, the relative frequency of CVD as the highest proportion of comorbidities with COVID-19 was varied from $9.91 \%$ to $10.22 \%$. It is remarkable, the relative frequency of asthma and the other CRD reached from 3.87 to $4.87 \%$ during these months. And, the percent of cancer comorbidity in hospitalized infected patients with COVID-19 considerably was increased from 1.00 to $2.18 \%$ (Fig. 1).

According to medical care monitoring center (MCMC) data analysis, CVD and diabetes were the most comorbidities in death cases of COVID-19. Across 3 months, CVD reported in died persons with COVID-19 as follow; first month $16.09 \%$, second month $16.81 \%$, and third month $16.59 \%$. Also, the proportion of diabetic persons in COVID-19 patients who died in hospital was considerable $(14.92 \%$ in first month and $16.68 \%$ in third month).

Comorbidity of asthma and other CRD had increasing trend in hospitalized died cases due to novel coronavirus across 90 days, and reached from 5.71 to $7.00 \%$. It is noticeable, from 20 Feb to 20 May 2020, comorbidity of cancer in COVID-19 death cases has been doubled and increased from 2.10 to $5.53 \%$ (Fig. 2).

\section{Conclusion}

According to our study, NCDs had a critical role in mortality rate of infected persons with COVID-19. Across 3 months, ascending trends of CVD, diabetes, CRD, and cancer's relative frequency in hospitalized confirmed cases of COVID-19 and occurred death in Iran is important issue that required special attention. It should be noted, this situation might be related to prevalence of mentioned diseases in Iran, and may be result of hospitalization rate. Anyway, we faced to complex situation.

In Italy, comorbidities including hypertension (69.2\%), type 2 diabetes $(31.8 \%)$, ischemic heart disease $(28.2 \%)$, chronic obstructive pulmonary disease $(16.9 \%)$, and cancer $(16.3 \%)$ reported in died hospital patients of COVID-19
Fig. 2 Relative frequency of the main NCDs as comorbidities in death cases in hospitalized COVID-19 confirmed cases. $C V D$ cardiovascular diseases, $C R D$ chronic respiratory diseases

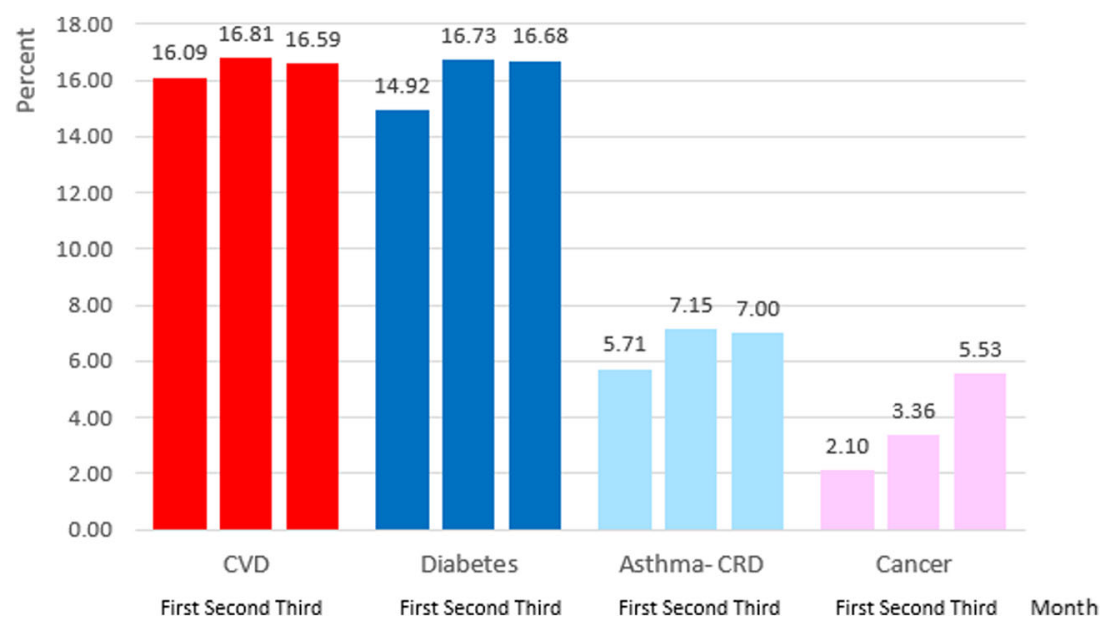


[29]. These measures in Italy have been higher than Iran based on our study. A study in Wuhan showed that the number of co-morbidities could be a significant predictor of mortality [11].

Therefore, the COVID-19 crisis might be intensified by the NCDs' comorbidities and would be effect on health, economy, and social development that needs urgent action. Based on the World Health Organization report on result of rapid assessment of service delivery for NCDs during the COVID19 pandemic, NCDs services disrupted in 120 countries [30]. In the more severe transmission phase of the COVID-19 pandemic (community transmission), disruption services for hypertension management, treatment of diabetes and its complications, cancer, and cardiovascular emergencies was occurred in $64 \%, 62 \%, 54 \%$, and $46 \%$ of countries, respectfully [30]. "Decrease in inpatient volume due to cancellation of elective care", "Closure of population-level screening programs", "Government or public transport lockdowns hindering access to the health facilities", and "NCD related clinical staff deployed to provide COVID-19 relief" were the main causes of NCDs service disruption [30]. Prioritize NCDs patients for COVID-19 testing, deliver essential NCDs medicine and equipment to home, use telemedicine, and special attention to COVID-19 cases with NCDs could be benefit for management this disease [31].

This is a critical time for all governments to adapt NCDs' national action plan to address the NCD-related policy and legislation gaps [32]. Implementation appropriate interventions in the COVID-19 pandemic to ensure access to care for people with NCDs, strengthen different levels of health care system through trained health care workers, and financial support could be reduced double burden of disease and promote appropriate response to this present critical situation.

Therefore we should address this situation in our national action plan for NCDs [33] and Iranian non communicable diseases committee (INCDC) policies [34] as high level multi-sectoral policy making statement by developing a national roadmap.

Acknowledgments We acknowledge statistic and information technology center of ministry of health for leading data collection of COVID-19 across the country, and all health workers for their effort in control of COVID-19 epidemic in Iran.

\section{Compliance with ethical standards}

Conflict of interest The authors declare that they have no conflict of interest.

\section{References}

1. WHO. WHO Coronavirus Disease (COVID-19) Dashboard. https://covid19.who.int/. 2020.
2. National Committee on COVID-19 Epidemiology MoHaME. http://corona.behdasht.gov.ir/files/site1/files/IRAN_COVID19 Factsheet_N.45_-13June_En.pdf. 2020.

3. Hunter DJ, Reddy KS. Noncommunicable diseases. N Engl J Med. 2013;369(14):1336-43.

4. GBD compare. Avaialble at: https://vizhub.healthdata.org/gbdcompare/.

5. Baradaran A, Ebrahimzadeh MH, Baradaran A, Kachooei AR. Prevalence of comorbidities in COVID-19 patients: a systematic review and meta-analysis. Arch Bone Jt Surg. 2020;8:247-55.

6. Chen QQ, Zheng ZC, Zhang C, Zhang XJ, Wu HJ, Wang JD, et al. Clinical characteristics of 145 patients with corona virus disease 2019 (COVID-19) in Taizhou, Zhejiang, China. Infection. 2020;48(4):543-51. https://doi.org/10.1007/s15010-020-01432-5.

7. Chen T, Wu D, Chen HL, Yan WM, Yang DL, Chen G, et al. Clinical characteristics of 113 deceased patients with coronavirus disease 2019: retrospective study. BMJ. 2020;368:m1295. https:// doi.org/10.1136/bmj.m1295.

8. Emami A, Javanmardi F, Pirbonyeh N, Akbari A. Prevalence of underlying diseases in hospitalized patients with COVID-19: a systematic review and meta-analysis. Arch Acad Emerg Med. 2020;8(1):e35.

9. Bansal M. Cardiovascular disease and COVID-19. Diabetes Metab Syndr. 2020;14(3):247-50.

10. Zheng YY, Ma YT, Zhang JY, Xie X. COVID-19 and the cardiovascular system. Nat Rev Cardiol. 2020;17(5):259-60. https://doi. org/10.1038/s41569-020-0360-5.

11. Ruan Q, Yang K, Wang W, Jiang L, Song J. Clinical predictors of mortality due to COVID-19 based on an analysis of data of 150 patients from Wuhan, China. Intensive Care Med. 2020;46(5):8468. https://doi.org/10.1007/s00134-020-05991-x.

12. Yang $\mathrm{C}$, Jin $\mathrm{Z}$. An acute respiratory infection runs into the most common noncommunicable epidemic - COVID-19 and cardiovascular diseases. JAMA Cardiol. 2020;5(7):743-4. https://doi.org/10. 1001/jamacardio.2020.0934.

13. Gupta R, Ghosh A, Singh AK, Misra A. Clinical considerations for patients with diabetes in times of COVID-19 epidemic. Diabetes Metab Syndr. 2020;14(3):211-2. https://doi.org/10.1016/j.dsx. 2020.03.002.

14. Brufsky A. Hyperglycemia, hydroxychloroquine, and the COVID19 pandemic. J Med Virol. 2020;92(7):770-5. https://doi.org/10. 1002/jmv.25887.

15. Maddaloni E, Buzzetti R. Covid-19 and diabetes mellitus: unveiling the interaction of two pandemics. Diabetes Metab Res Rev. 2020: e33213321. https://doi.org/10.1002/dmrr.3321.

16. Wang XH, Fang XX, Cai ZX, Wu XT, Gao XT, Min JX, et al. Comorbid chronic diseases and acute organ injuries are strongly correlated with disease severity and mortality among COVID-19 patients: a systemic review and meta-analysis. Research (Wash D C). 2020;2020:2402961. https://doi.org/10.34133/2020/2402961.

17. Wu Z, McGoogan JM. Characteristics of and important lessons from the coronavirus disease 2019 (COVID-19) outbreak in China: summary of a report of 72314 cases from the Chinese Center for Disease Control and Prevention. JAMA. 2020;323(13): 1239-42. https://doi.org/10.1001/jama.2020.2648.

18. Wang WQ, Lu JL, Gu WQ, Zhang YF, Liu JM, Ning G. Care for diabetes with COVID-19: advice from China. J Diabetes. 2020;12(5):417-9. https://doi.org/10.1111/1753-0407.13036.

19. Trapani D, Marra A, Curigliano G. The experience on coronavirus disease 2019 and cancer from an oncology hub institution in Milan, Lombardy region. Eur J Cancer. 2020;132:199-206.

20. Desai A, Sachdeva S, Parekh T, Desai R. COVID-19 and cancer: lessons from a pooled meta-analysis. JCO Glob Oncol. 2020;6: 557-9. https://doi.org/10.1200/GO.20.00097. 
21. Wang H, Zhang L. Risk of COVID-19 for patients with cancer. Lancet Oncol. 2020;21(4):e181. https://doi.org/10.1016/S14702045(20)30149-2.

22. Sidaway P. COVID-19 and cancer: what we know so far. Nat Rev Clin Oncol. 2020;17(6):336. https://doi.org/10.1038/s41571-0200366-2.

23. Liang W, Guan W, Chen R, Wang W, Li J, Xu K, et al. Cancer patients in SARS-CoV-2 infection: a nationwide analysis in China. Lancet Oncol. 2020;21(3):335-7.

24. Tal-Singer R, Crapo JD. COPD at the time of COVID-19: a COPD Foundation perspective. Chronic Obst Pulm Dis. 2020;7(2):73-5.

25. Zhao Q, Meng M, Kumar R, Wu Y, Huang J, Lian N, et al. The impact of COPD and smoking history on the severity of Covid-19: a systemic review and meta-analysis. J Med Virol. 2020. https://doi. org/10.1002/jmv.25889.

26. Alqahtani JS, Oyelade T, Aldhahir AM, Alghamdi SM, Almehmadi M, Alqahtani AS, et al. Prevalence, severity and mortality associated with COPD and smoking in patients with COVID19: a rapid systematic review and meta-analysis. PLoS One. 2020;15(5):e0233147. https://doi.org/10.1371/journal.pone. 0233147.

27. Kaye L, Theye B, Smeenk I, Gondalia R, Barrett MA, Stempel DA. Changes in medication adherence among patients with asthma and COPD during the COVID-19 pandemic. J Allergy Clin Immunol Pract. 2020;8(7):2384-5.

28. National Committee on COVID-19 Epidemiology, Ministry of Health and Medical Education, IR Iran. Daily situation report on coronavirus disease (COVID-19) in Iran; march 23, 2020. Arch Acad Emerg Med. 2020;8(1):e36.
29. Kluge HHP, Wickramasinghe K, Rippin HL, Mendes R, Peters DH, Kontsevaya A, et al. Prevention and control of noncommunicable diseases in the COVID-19 response. Lancet. 2020;395(10238):1678-80.

30. Rapid assessment of service delivery for NCDs during the COVID19 pandemic. Available at: https://www.who.int/publications/m/ item/rapid-assessment-of-service-delivery-for-ncds-during-thecovid-19-pandemic. Last Access: 29 May 2020.

31. Deng SQ, Peng HJ. Characteristics of and public health responses to the coronavirus disease 2019 outbreak in China. J Clin Med. 2020;9(2). https://doi.org/10.3390/jcm9020575.

32. Tin STW, Vivili P, Na'ati E, Bertrand S, Kubuabola I. Insights in public health: COVID-19 special column: the crisis of noncommunicable diseases in the Pacific and the coronavirus disease 2019 pandemic. Hawaii J Health Soc Welf. 2020;79(5):147-8.

33. Peykari N, Hashemi H, Dinarvand R, Haji-Aghajani M, Malekzadeh R, Sadrolsadat A, et al. National action plan for noncommunicable diseases prevention and control in Iran; a response to emerging epidemic. Journal of Diabetes \& Metabolic Disorders. 2017;16(1):3. https://doi.org/10.1186/s40200-017-0288-4.

34. Peykari N, Larijani B. A multi-sectoral approach to combatting non-communicable diseases: Iran's experience. Journal of Diabetes \& Metabolic Disorders. 2019;18(2):719-20.

Publisher's note Springer Nature remains neutral with regard to jurisdictional claims in published maps and institutional affiliations. 